\section{IN THE NEWS}

Grey matter matters

'Grey matter' has become

synonymous with intelligence, yet to neuroscientists it is simply the part of the cortex and spinal cord that houses nerve cell bodies. However the authors of a new study hope to change our minds, by showing that the grey matter might really be, as the Independent (UK, 5

November) delicately puts it, "the intellectual stuffing of the brain".

Paul Thompson's team at the University of California showed that the brains of identical twins are more similar in grey matter content than those of non-identical twins, indicating that this is controlled genetically. As the New York Times (5 November) reports, "the quantity of gray matter in the frontal lobes was under particularly tight control, as was ... Wernicke's area, which is central to language". Interestingly, people with more grey matter tended to perform better in IQ tests.

Alzheimer's expert Bruce Miller describes the work as "an exciting study that starts to show there are some brain areas in which there are very significant genetic influences on structure" (New York

Times, 5 November). On the question of IQ tests, behavioural scientist Robert Plomin believes that "[grey matter content] is actually something real in the brain that correlates with people's ability to solve these sorts of problems" (Independent)

Separating cause from effect might prove trickier. As Plomin points out, "people with a stronger motivation, say, might exercise their brains harder and develop a high density of neurons" (New York Times). So, brain scanning is unlikely to replace more traditional methods of intelligence testing any time soon. Thompson stresses that the volume of grey matter does not necessarily predict intelligence, and in any case "It would be remiss to use technology developed for disease for those types of goals" (New York Times)

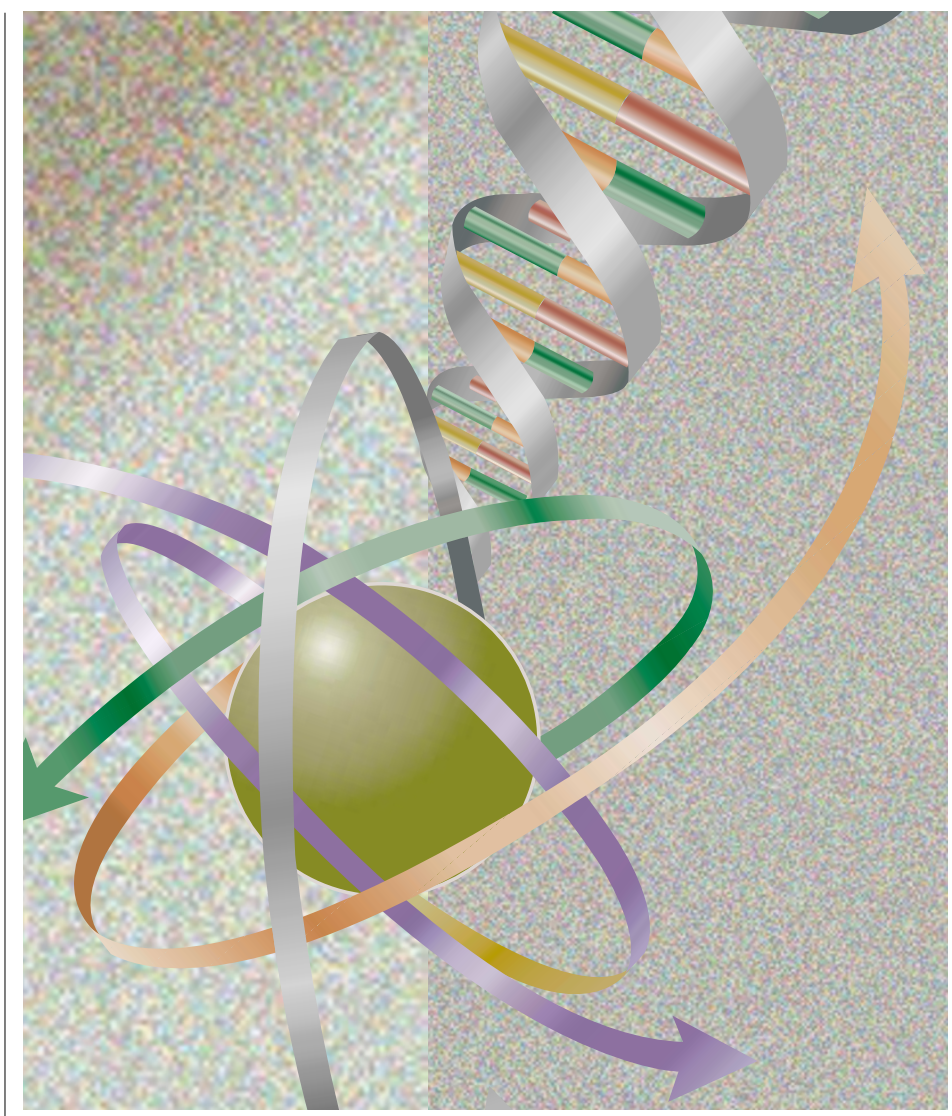

GENE THERAPY

\title{
DNA delivery
}

Successful, safe gene therapy is something of a Holy Grail for researchers. Delivering an exogenous gene into the targeted cells, and ensuring that it will be expressed there, are fraught with difficulties. Viruses are a promising vector for the delivery of a therapeutic gene into a patient's cells, but bring with them a new set of problems; for example, how to avoid an inflammatory or immune reaction to the virus. An alternative, non-viral delivery method is beginning to show potential.

Shi et al. have previously shown that 'pegylated' liposomes - coated with strands of polyethylene glycol can be used as nanocontainers to carry DNA from the bloodstream into various tissues, including the brain, in rats. In that experiment, the liposomes were conjugated with a monoclonal antibody against the transferrin receptor, and the exogenous gene

\section{SNARE-free zone}

The SNARE hypothesis was first postulated nearly a decade ago as a mechanistic account of membrane fusion. In its earliest form, this hypothesis stated that SNARE proteins present in the acceptor and donor membranes mediate the specificity of the interaction preceding fusion. The hypothesis was later modified to propose that SNARE proteins are actually involved directly in the fusion reaction. At synapses, the main fusion event takes place during transmitter release, as synaptic vesicles fuse with the presynaptic membrane. The SNARE hypothesis was quickly invoked to explain this process, and suggestions of relevant SNARE proteins were rapidly put forward from the growing list of molecules present at synaptic contacts. These developments led to the optimistic view that the intimate details of synaptic exocytosis would soon be unveiled. Now, almost 10 years later, some of our basic questions on this phenomenon remain unanswered, including that of the actual role of SNAREs in fusion. Are SNAREs the molecular components of the fusion pore? Do they act as catalysts of the fusion reaction, or are they only involved in setting up its specificity? Reporting in Science, Schoch et al. argue that SNAREs are not necessary for the fusion step per se, but are required instead for stabilizing fusion intermediates.

Schoch et al. generated mice that lack synaptobrevin 2 - a SNARE protein of synaptic vesicles - and studied the characteristics of transmitter release in neurons cultured from the forebrains of these animals. They found that synaptic vesicle exocytosis was markedly reduced, regardless of whether it was spontaneous or evoked by changes in osmolarity or calcium influx. However, not all forms of exocytosis were equally affected; spontaneous and osmolarity-evoked release were 10-fold lower than in wild-type neurons, whereas calcium-evoked release was reduced by 100 -fold. Changes in osmolarity and calcium influx affect the same population of vesicles. So, if synaptobrevin were involved directly in fusion, it would be hard to account for this difference. The authors therefore argue that synaptobrevin is not required for fusion per se, but is necessary to achieve a normal rate of fusion on stimulation. In other words, they propose that SNARE proteins stabilize transition states that exist before vesicles are responsive to calcium, and that although they do not participate in actual fusion, they limit the rate at which fusion can occur. It will now be crucial to 